\title{
Normovolemic hemodilution and the heart
}

\author{
Philippe Van der Linden MD PhD, ${ }^{*}$ Stefan De Hert MD PhD $\dagger$
}

$\mathrm{M}$ AINTENANCE of adequate tissue oxygenation during acute isovolemic hemodilution depends on physiologic adjustments occurring both at the systemic and the microcirculatory levels, which result in increased tissue blood flow and oxygen extraction. ${ }^{1}$ These mechanisms allow the maintenance of tissue oxygen balance until the hematocrit falls to about 10 to $12 \% .^{2}$

At the level of the heart, oxygen extraction is already nearly maximal under resting conditions. ${ }^{3}$ Therefore, maintenance of myocardial oxygen delivery during isovolemic hemodilution depends essentially on the increase in coronary blood flow. This is achieved by a reduction in coronary vascular resistance related to the decreased blood viscosity but also to specific coronary vasodilation as demonstrated by analysis of vascular hindrance (vascular resistance/blood viscosity). ${ }^{3}$ In addition, isovolemic hemodilution is associated with an increased cardiac work resulting in an augmentation of myocardial oxygen demand. Indeed, heart rate, ${ }^{4}$ but also possibly myocardial contractility, ${ }^{5}$ has been shown to increase during isovolemic hemodilution. When the hematocrit is reduced to 10 to $12 \%$, myocardial oxygen consumption more than doubles. ${ }^{6}$ In these conditions, coronary vasodilatation is near maximal. Below a hematocrit of $10 \%$, coronary blood flow can no longer match the increased myocardial oxygen demand and ischemia develops, ultimately resulting in cardiac failure. This is in accordance with experimental data showing a decrease in systemic oxygen uptake at hematocrit values close to $10 \%{ }^{7}$

As maximal coronary blood flow is not influenced by hemodilution, the coronary reserve (the ratio between maximal coronary blood flow and resting coronary blood flow) is significantly reduced during isovolemic anemia. Geha ${ }^{8}$ demonstrated in intact dogs a $50 \%$ reduction in the coronary flow reserve when hematocrit is decreased from 43 to $20 \%$. This indicates the vulnerability of the heart during anemia, especial- ly if coronary artery disease (decreased maximal coronary blood flow) or higher work demands on the myocardium should co-exist. The lowest tolerable hematocrit in coronary artery disease patients is not known but experimental data in animals with extrinsically applied coronary stenosis have demonstrated a significant increase in the value of the hematocrit associated with the development of cardiac failure. ${ }^{9}$ Based on a theoretical analysis, Kettler ${ }^{10}$ estimated that coronary artery disease patients may tolerate some degree of hemodilution intraoperatively but will require a higher hematocrit in the early postoperative period to meet the increased tissue, and especially cardiac, oxygen demand. This has been confirmed by the study of Nelson et al.," ${ }^{11}$ showing that a postoperative hematocrit below $28 \%$ was associated with increased myocardial ischemic episodes and morbid cardiac events. Cardiovascular disease patients with a lower preoperative hematocrit have an increased risk of death when compared to non-cardiovascular disease patients with the same preoperative hematocrit. ${ }^{12}$

In the present issue, Tonkovic et al. ${ }^{13}$ evaluated the hemodynamic response to moderate dobutamine doses in hemodiluted coronary artery disease patients scheduled for off-pump surgery. Patients were hemodiluted to a target hemoglobin of either 95 to $105 \mathrm{~g} \cdot \mathrm{L}^{-1}[\bmod -$ erate acute normovelemic hemodilution $(\mathrm{ANH})]$ or 75 to $85 \mathrm{~g} \cdot \mathrm{L}^{-1}$ (severe ANH). Dobutamine infusion at a rate of $5 \mu \mathrm{g} \cdot \mathrm{kg}^{-1} \cdot \mathrm{min}^{-1}$ was associated with a significant increase in cardiac index in patients undergoing moderate $\mathrm{ANH}$ but not in patients undergoing severe $\mathrm{ANH}$.

These observations merely confirm the physiological concepts developed above. Dobutamine similarly increased myocardial oxygen demand in both groups, as is obvious from the increase in heart rate and mean arterial pressure. Indeed, the rate pressure product is frequently used as a clinical estimate of myocardial oxygen demand. ${ }^{14}$ However, it is likely that in patients undergoing the more profound hemodilution protocol, coronary flow reserve was nearly exhausted so that

From the Departments of Anesthesiology, CHU Brugmann-HUDERF, * Brussels; and the U.Z. Antwerpen, $†$ Antwerpen, Belgium. Address correspondence to: Dr. Philippe Van der Linden, Department of Anesthesiology, CHU Brugmann - HUDERF, 4 place van Gehuchten, B-1020 Brussels. Phone: 32-2-477-3996; Fax: 32-2-477-3345; E-mail: philippe.vanderlinden@chu-brugmann.be 
their myocardium was unable to sustain the additional load imposed by dobutamine. Not only did cardiac output not change, but stroke volume index and left ventricular stroke work index decreased in response to the $B$-adrenergic agent. Although the authors did not observe ischemic changes on the electrocardiogram, the deterioration of myocardial function strongly suggests the occurrence of myocardial oxygen supply demand imbalance.

Normovolemic hemodilution represents a safe blood conservative technique as far as its limitations, especially coronary artery disease, are recognized and respected.

\section{Le cour et l'hémodilu- tion normovolémique}

Le maintien d'une oxygénation tissulaire adéquate pendant l'hémodilution isovolémique aiguë dépend d'ajustements physiologiques systémiques et microcirculatoires dont le résultat est une augmentation du débit sanguin tissulaire et de l'extraction d'oxygène. ${ }^{1}$ Ces mécanismes permettent de maintenir l'équilibre de l'oxygénation tissulaire jusqu'à ce que l'hématocrite baisse aux environs de 10 à $12 \% .^{2}$

Au niveau du cœur, l'extraction d'oxygène est déjà presque maximale au repos. ${ }^{3}$ Par conséquent, le maintien de l'apport d'oxygène myocardique pendant l'hémodilution isovolémique dépend essentiellement de l'augmentation du débit coronaire. Ce qui s'obtient par une réduction de la résistance vasculaire coronaire reliée à la baisse de la viscosité du sang, mais aussi à une vasodilatation coronaire spécifique comme l'a démontré l'analyse de l'entrave vasculaire (résistance vasculaire/viscosité sanguine). ${ }^{3}$ De plus, l'hémodilution isovolémique est associée à une hausse du travail cardiaque qui va provoquer une augmentation de la demande d'oxygène myocardique. En effet, on a montré que la fréquence cardiaque, ${ }^{4}$ mais aussi peut-être la contractilité myocardique, ${ }^{5}$ augmentait pendant l'hémodilution isovolémique. Quand l'hématocrite est à 10 à $12 \%$, la consommation d'oxygène myocardique est plus que doublée. ${ }^{6}$ Dans ces conditions, la vasodilatation coronaire est presque maximale. Pour un hématocrite sous les $10 \%$, le débit coronarien ne peut plus répondre à la demande accrue d'oxygène myocardique et l'ischémie se développe, provoquant à la limite une insuffisance cardiaque. Ces données correspondent à celles d'expériences qui montrent une baisse de la consommation générale d'oxygène pour des valeurs d'hématocrite proches de $10 \%{ }^{7}$

Le débit coronarien maximal n'étant pas modifié par l'hémodilution, la réserve coronaire (le rapport entre le débit coronaire maximal et le débit coronaire au repos) est significativement réduite pendant l'anémie isovolémique. Geha ${ }^{8}$ a démontré chez des chiens intacts une réduction de $50 \%$ de la réserve coronaire quand l'hématocrite est passé de 43 à $20 \%$. Cela indique la vulnérabilité du cœur pendant l'anémie, surtout si une cardiopathie ischémique (baisse du débit coronaire maximal) existe ou si un plus grand effort est exigé du myocarde. Le plus bas niveau d'hématocrite tolérable en cas de cardiopathie ischémique n'est pas connu, mais des expériences sur des animaux, chez qui on a provoqué une sténose coronaire extrinsèque, ont démontré une hausse significative de la valeur de l'hématocrite associée au développement de l'insuffisance cardiaque. ${ }^{9}$ En s'appuyant sur une analyse théorique, Kettler ${ }^{10}$ a estimé que les patients atteints de cardiopathie ischémique pouvaient tolérer un certain degré d'hémodilution peropératoire, mais qu'un hématocrite plus élevé au début de la période postopératoire était nécessaire pour répondre à la demande accrue d'oxygène tissulaire et, spécialement, cardiaque. Nelson et coll., ${ }^{11}$ le confirment en montrant qu'un hématocrite postopératoire sous $28 \%$ est associé à une augmentation des épisodes d'ischémie myocardique et des événements cardiaques morbides. Les patients atteints de maladie cardiovasculaire qui présentent un hématocrite préopératoire faible risquent plus de mourir que ceux qui ont le même hématocrite préopératoire sans maladie cardiovasculaire. ${ }^{12}$

Dans le présent numéro, Tonkovic et coll..$^{13}$ ont évalué la réponse hémodynamique à des doses modérées de dobutamine chez des patients devant subir une opération de pontages coronaire à cœur battant. Les patients étaient sous hémodilution selon un taux cible de 95 à $105 \mathrm{~g} \cdot \mathrm{L}^{-1}$ [hémodilution normovolémique aiguë modérée (HNA)] ou de 75 à $85 \mathrm{~g} \cdot \mathrm{L}^{-1}$ (HNA sévère). La perfusion de dobutamine à $5 \mu \mathrm{g} \cdot \mathrm{kg}^{-1} \cdot \mathrm{min}^{-1}$ a été associée à une augmentation significative de l'index cardiaque chez les patients soumis à une HNA modérée, mais non chez les patients sous HNA sévère.

Ces observations ne font que confirmer les concepts physiologiques élaborés plus haut. La dobutamine a augmenté la demande d'oxygène myocardique de la même façon chez les patients des deux groupes comme l'indique la hausse de la fréquence cardiaque et de la tension artérielle moyenne. Il est vrai que le produit tension-fréquence est souvent utilisé comme 
estimation clinique de la demande en oxygène myocardique. ${ }^{14}$ Toutefois, il est possible que chez les patients soumis à un protocole d'hémodilution plus marquée, la réserve coronarienne était presque épuisée de sorte que leur myocarde ne pouvait supporter la charge supplémentaire imposée par la dobutamine. Non seulement le débit cardiaque n'a pas changé, mais l'index du volume d'éjection et l'index du travail systolique ventriculaire gauche ont diminué en réponse au médicament $ß$-adrénergique. Bien que les auteurs n'aient pas observé de changement ischémique à l'électrocardiogramme, la détérioration de la fonction myocardique suggère fortement l'occurrence d'un déséquilibre entre la demande et l'apport d'oxygène myocardique.

L'hémodilution normovolémique est une technique sûre d'économie de sang en autant que ses limites, spécialement la cardiopathie ischémique, soient reconnues et respectées.

\section{References}

1 Van der Linden P. The physiology of acute isovolaemic anaemia. Acta Anaesthesiol Belg 2002; 53: 97-103.

2 Chapler CK, Cain SM. The physiologic reserve in oxygen carrying capacity: studies in experimental hemodilution. Can J Physiol Pharmacol 1986; 64: 7-12.

3 Jan KM, Chien S. Effect of hematocrit variations on coronary hemodynamics and oxygen utilization. Am J Physiol 1977; 233: H106-13.

4 Weiskopf RB, Feiner J, Hopf H, et al. Heart rate increases linearly in response to acute isovolemic anemia. Transfusion 2003; 43: 235-40.

5 Habler OP, Kleen MS, Podtschaske AH, et al. The effect of acute normovolemic hemodilution (ANH) on myocardial contractility in anesthetized dogs. Anesth Analg 1996; 83: 451-8.

6 von Restorff W, Hofling B, Holtz J, Bassenge E. Effect of increased blood fluidity through hemodilution on coronary circulation at rest and during exercise in dogs. Pflugers Arch 1975; 357: 15-24.

7 Cain SM. Oxygen delivery and uptake in dogs during anemic and hypoxic hypoxia. J Appl Physiol 1977; 42: 228-34.

8 Geha AS. Coronary and cardiovascular dynamics and oxygen availability during acute normovolemic anemia. Surgery 1976; 80: 47-53.

9 Levy PS, Kim SJ, Eckel PK, et al. Limit to cardiac compensation during acute isovolemic hemodilution: influence of coronary stenosis. Am J Physiol 1993; 265: H340-9.

10 Kettler D. "Permissive anaemia" compared with blood transfusion in patients with cardiac disease: another point of view. Curr Opin Anesth 1994; 7: 1-4.
11 Nelson AH, Fleisher LA, Rosenbaum SH. Relationship between postoperative anemia and cardiac morbidity in high-risk vascular patients in the intensive care unit. Crit Care Med 1993; 21: 860-6.

12 Carson JL, Duff A, Poses RM, et al. Effect of anaemia and cardiovascular disease on surgical mortality and morbidity. Lancet 1996; 348: 1055-60.

13 Tonkovic D, Husedzinovic I, Barisin S, et al. Hemodynamic response to moderate dobutamine dose in OPCAB during acute normovolemic hemodilution. Can J Anesth 2005; 52: 214-5.

14 Crystal GJ. Principles of cardiovascular physiology. In: Estafanous FG, Barash PG, Reves JG (Eds). Cardiac Anesthesia: Principles and Clinical Practice. Philadelphia: Lippincott-Williams \& Wilkins; 2001: 37-57. 